\title{
Power Dispatch Combining Meteorological Forecast and Dynamic Game Model in Multivariate Distributed Power Generation Systems
}

\author{
Long-Yi Chang $^{1 *}$ and Shiu-Fu Lin ${ }^{2}$ \\ ${ }^{1}$ Department of Electrical Engineering, National Chin-Yi University of Technology, \\ No. 57, Sec. 2, Zhongshan Rd., Taiping District, Taichung 41170, Taiwan \\ ${ }^{2}$ Department of Electrical Engineering, National Chin-Yi University of Technology, \\ No. 57, Sec. 2, Zhongshan Rd., Taiping District, Taichung 41170, Taiwan
}

(Received June 22, 2020; accepted December 10, 2020)

Keywords: distributed energy, demand bidding, demand response, power dispatching, DGM

We propose a system of distributed energy that combines a dynamic game model (DGM) with the Central Weather Bureau's weather data, and apply it to the power dispatch of a distributed grid. A programmable logic controller (PLC) and human-machine interface (HMI) are used to build the distributed grid for dispatching renewable energy. Moreover, the weather overview for 3 days is downloaded by using MATLAB/Simulink and the Python programming language to link to the weather forecast data, and the power generation on the next day is estimated on the basis of the weather data to dispatch the distributed energy according to the time-of-use power price and management plan announced by the power company. In addition, the weather data may change owing to the changeability of the weather. Therefore, this system can, at any time, obtain the latest weather data, apply the DGM, and dispatch the stored distributed energy in accordance with the power company's demand bidding measures, so that the power system can achieve the most economic power when there is high demand. It was found that the managed dispatch of all the power supplies and loads had optimal efficiencies, and the power company's reserve capacity was increased to achieve optimal power dispatch.

\section{Introduction}

In recent years, with the temperature rise in summers, the electricity consumption in Taiwan has risen dramatically. As a result, Taiwan Power Company has been short of power supply, with the estimated operating reserve ratio having become increasingly low. With the increase in environmental awareness, clean, pollution-free, and inexhaustible energy is urgently needed. Hence, the purposes of this paper are the development and integration of renewable energy. As a solution for all governments to issues such as world energy, global warming, air pollution, and urban living, the applications of distributed grids are gradually expanding. These grids use regional energy management dispatch and distribution through the real-time information analysis of energy-related data to achieve energy conservation, carbon reduction, and improved power efficiency.

*Corresponding author: e-mail: lychang@ncut.edu.tw https://doi.org/10.18494/SAM.2021.3021 
Most traditional power generation systems are centralized, transferring power from power plants to the regions where power is needed. As the power demand increases, the operation and maintenance costs increase, and it becomes difficult to meet user needs for stability and safety. Taiwan suffered a power cut crisis from July 29, 2017 to August 14, 2017; the power transmission tower of Heping power plant collapsed on July 29, 2017 due to Typhoon Nesat, so power could not be transmitted outward. Taiwan Power Company immediately lost about 1.3 GW of its reserve capacity, about $4 \%$ of the operating reserve ratio, and it was only just possible to avoid a power cut. Even with ample operating reserves, if such serious failures or emergencies occur in the power transmission and distribution system, power plants will be unable to transmit power, leading to supply problems and power cuts. The vulnerability and instability of power grids are also revealed in natural disasters and emergencies. Compared with centralized power grids, distributed grids have advantages such as a high energy utilization ratio, low pollution, and small line loss. The main reason why distributed grids were not applied in the past is that the power could not be adjusted in a timely manner with the peak demand; owing to the communication technology and infrastructure technology in the past, the power needed to be determined one day in advance. Moreover, renewable energy used to be more unstable and cause many difficulties in power grid operation and dispatch, which greatly affected the quality and reliability of the power supply in power grids.

Nowadays, with the advanced technology and rapid progress of energy information technology, smart electricity meters have been developed and become a key infrastructure of distributed grids. The advanced metering infrastructure (AMI) is growing in popularity, allowing users to know their power consumption and power prices through smart electricity meters and online information systems. ${ }^{(1,2)}$ Moreover, the cost of managing power demand is greatly reduced. The common strategies in performing demand response (DR), including time of use (TOU), real-time pricing (RTP), critical-peak pricing (CPP), peak time rebate (PTR), and system peak response transmission tariff, provide a basis for users to determine whether to consume power at present by controlling their consumption based on the price. ${ }^{(3)}$ DR refers to customer selling (demand) power back to the system in response to some signal (i.e., a price emergency call), or refers to the capacity of an electricity customer to reduce their consumption as the price rises on an hourly basis in wholesale markets or to reduce their consumption in response to emergency calls for curtailment or to reduce the load to forestall the need to implement rolling blackouts. The ways of implementing DR can be roughly divided into the demand side and supply side. The demand side refers to client management; if there is high demand, the power users are made to reduce their load. The method can be active or passive, and the main difference between them is whether the users have the right to participate in the DR plan. In the active DR, the power company announces the electricity charge deduction standard on the bidding platform at fixed times to make the users change their power consumption patterns, which is also called demand bidding. In the passive DR, the power company directly reduces the load of power users when a DR is needed, where the most common way is direct load control (DLC). ${ }^{(4)}$ In this study, through the incentive of tariff discount, users can reduce peak load power consumption, and the concept of "time price" is used to change the user's electricity consumption habits. Part of the power demand is transferred 
from the period of higher price to the period of lower price. As a result, the user is rewarded by a reduction in the cost of electricity provided by the power company, and the power company can also reduce the power generation cost and increase reserve capacity during peak demand.

\section{Research Method}

A dynamic game model (DGM) can be applied to a smart grid. ${ }^{(4)}$ The DR of users in each hour is predicted, and the DGM is used to allocate auxiliary service power according to the user DR and interruptible power. ${ }^{(5,6)}$

The energy of distributed grids is a diverse system and the renewable energy output fluctuates greatly, so the energy dispatch system must have sufficient capacity, load control, and voltage regulation by adjusting the power for the users according to the operation status. Therefore, in this study, a weather forecasting model is added to estimate the power generation through the weather data and determine whether a DGM needs to participate in the DR.

The distributed energy in this study includes solar energy, wind energy, fuel cells, backup diesel generators, and storage batteries, among which solar energy accounts for the largest proportion. Hence, it can be determined from the amount of solar energy generation whether to participate in the DR and whether to turn on generators or reduce the load for energy saving, carbon emission reduction, and electricity cost reduction.

\subsection{Solar energy generation}

The principle of solar energy generation forecasting is usually based on weather forecast data or actually measured weather data, and suitable forecast models and algorithms have been established to forecast solar power generation for some time in the future by combining them with factors related to geographical location. There are many types of methods to forecast solar power generation, which can be divided into direct and indirect forecast methods according to the forecast process. The direct forecast method is to forecast the power generation under the weather conditions for some time in the future, and this method is based on historical data and is also called data statistics. The indirect forecast method is to indirectly forecast the power generation of a solar energy system by forecasting the sunlight radiation intensity received by the ground or solar energy components. ${ }^{(7)}$

Solar power generation is related to factors such as sunlight intensity, temperature, weather, and geographical location, among which the meteorological factors including air temperature, humidity, and sunlight radiation are closely related to power generation. Figures 1(a) and 1(b) show the correlation between hourly power generation and temperature and humidity, respectively, indicating that the daily power generation is positively related to temperature and negatively related to humidity. Hence, air temperature and humidity were selected in this paper as the parameters of the power generation forecast model. The forecast model is given by Eq. (1), where $R H$ means humidity, $T$ means air temperature, and $S_{\text {solar }}$ means the forecast solar power generation. 




(a)

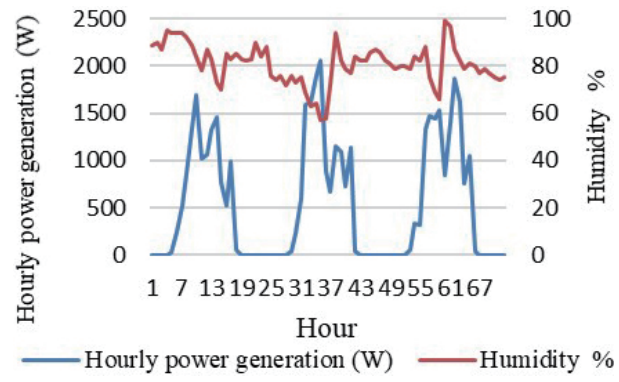

(b)

Fig. 1. (Color online) (a) Correlation between solar power generation and air temperature and (b) correlation between solar power generation and humidity.

$$
\begin{gathered}
\alpha=R H \times T \\
S_{\text {solar }}=\left\{\begin{array}{cc}
W d \leq 1000, & \alpha \geq 900 \\
1000<W d<2000 & 400<\alpha<900 \\
W d \geq 2000, & \alpha \leq 400
\end{array}\right.
\end{gathered}
$$

\subsection{Wind energy generation}

Two main models are used in the prediction of wind energy generation, namely, physical and statistical models, and both of them are similar to the models used for solar energy prediction. The physical model is used to simulate the prediction of a wind field based on the results of a numerical weather forecast, using mesoscale or microscale models. The statistical model is used to analyze the correlation between the wind field and the numerical weather forecast in the historical data and to forecast the wind-power generation within a certain period of time in the future, in the same way that the data statistics of solar energy are used for predictions and analyses based on big data. ${ }^{(8)}$

In wind-power generation, the blades of the wind generators are driven mainly by wind to generate electricity. In this paper, the hourly wind speed was established on the basis of the average wind speed of the daily meteorological data, as shown in Fig. 2(a). The wind-power generation prediction model [Eq. (2)] was established after the wind speed was calculated from the minimum wind speed in Table 1 to predict the hourly power generation $S_{\text {wind }}$ of a wind generator, where $\rho$ is the air density. Normally, the dry air density is about $1.29 \mathrm{~kg} / \mathrm{mm}^{3}$, so 1.29 was taken as $\rho$ in the calculation. $R$ is the radius of the wind generator blade in meters. In this paper, the specifications of a wind generator are as follows: the energy usage is $2 \mathrm{~kW}$, the rotation radius is $1.25 \mathrm{~m}$, the wind speed is $V_{W}$, and the daily power generation of the wind generator is shown in Fig. 2(b).

$$
S_{\text {wind }}=0.5 \times \rho \times \pi \times R^{2} \times V_{W}^{3}
$$

Finally, the total power capacity of renewable energy is given by

$$
S_{\text {renew }}=S_{\text {solar }}+S_{\text {fuel }}+S_{\text {wind }}+S_{\text {battery. }}
$$






(a)

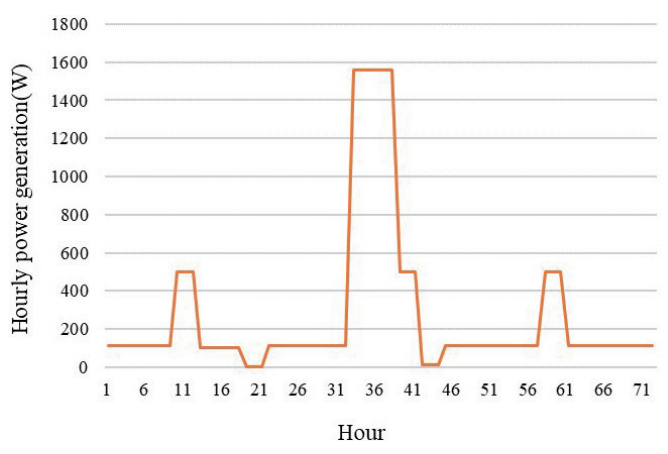

(b)

Fig. 2. (Color online) (a) Hourly wind speed and (b) hourly power generation.

Table 1

Beaufort scale.

\begin{tabular}{lc}
\hline Beaufort number & Wind speed $(\mathrm{m} / \mathrm{s})$ \\
\hline 0 & $0.3 \downarrow$ \\
1 & $0.3-1.5$ \\
2 & $1.6-3.3$ \\
3 & $3.4-5.4$ \\
4 & $5.5-7.9$ \\
5 & $8.0-10.7$ \\
6 & $10.8-13.8$ \\
7 & $13.9-17.1$ \\
8 & $17.2-20.7$ \\
\hline
\end{tabular}

\subsection{Establishment of DGM}

Under the condition of maximum user benefit, we first estimated the demand for the next day and the power that can be cut. Then the generating capacities of all units, the generating costs of all units, and the operating reserve were considered to find the optimal dispatch for units when performing the DR. According to the demand bidding management measures of Taiwan Power Company, the power consumption is reduced for 2 or $4 \mathrm{~h}$ per day, the peak hours are from 10 am to $12 \mathrm{pm}$ and from $1 \mathrm{pm}$ to $5 \mathrm{pm}$, and the solar power generation is greatest from 10 am to $3 \mathrm{pm}$. Hence, in this study, the time during which the DR plan was carried out was divided into two periods: 10 am to $12 \mathrm{pm}$ and $1 \mathrm{pm}$ to $3 \mathrm{pm}$, with $4 \mathrm{~h}$ of reducing power consumption, $h=10: 00,11: 00,13: 00,14: 00$ ( $h$ represents time). The capacity required by the distributed power supply during period $h, S_{D G, h}$, can be obtained from Eq. (4), where $S_{p, h}$ is the forecast demand and $S_{i, h}$ is the adjustable load reduction demand. ${ }^{(4)}$

$$
S_{D G, h}=S_{p, h}-S_{i, h}-S_{l, h}
$$

After the demand was calculated, the probability of participating in (cooperative) DR was estimated as 


$$
\begin{gathered}
D R_{h}=\exp \left[-1 \times\left(\frac{1-S_{l, h} / S_{p, h}}{0.5}\right)^{2}\right], D R_{h} \in[0,1] \\
D R_{h}^{\prime}=\left(1-D R_{h}\right) .
\end{gathered}
$$

$S_{p, h}$ can be used to obtain the DR state according to the forecast demand, which is expressed by the attribution degree from 0 to 1 . A value equal to or close to 1 indicates high willingness to participate in DR. Alternatively, if a decrease in the inducement value is announced in the previous hour, the willingness to participate decreases and the test value of Eq. (5) decreases. If the willingness to participate is low (non-cooperative), Eq. (6) is equal to or close to 1. In other words, $D R_{h}^{\prime}$ is the similarity detection of participation in DR, and users' willingness to participate is shown in Fig. 3(a). Using Eq. (7), we obtain the membership function of distributed energy resources. The requirement on the distributed energy resources is shown in Fig. 3(b).

$$
D R_{D G, h}^{\text {renew }}=\left\{\begin{array}{cc}
1, & 0 \leq S_{D G, h} \leq \frac{S_{\text {renew }}}{4} \\
\exp \left(\frac{-1}{2} \times\left(\frac{S_{D G, h}-S_{\text {renew }} / 4}{S_{\text {renew }} / 16}\right)^{2}\right), & S_{D G, h}>\frac{S_{\text {renew }}}{4}
\end{array}\right.
$$

The sum of the distributed energy capacities, $S_{D G}=S_{p, h}-S_{i, h}, h=10: 00,11: 00,13: 00,14: 00$, is used in the DGM to find the best profit distribution. The distributed energy resources are determined by the degree of participation to achieve the balance between supply and demand in the DGM. ${ }^{(9,10)}$ According to the users' DR and the requirement on distributed power resources, the users' DR can be divided into two strategies: (1) extent of willingness (participation); (2) extent of unwillingness (nonparticipation). The requirement on distributed power resources

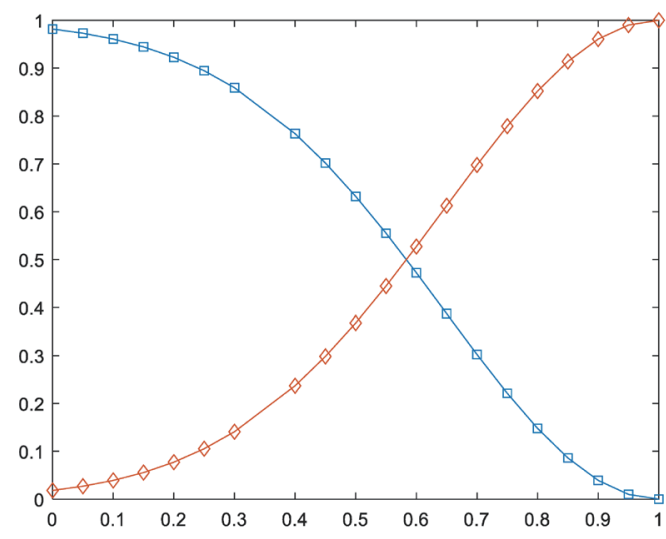

(a)

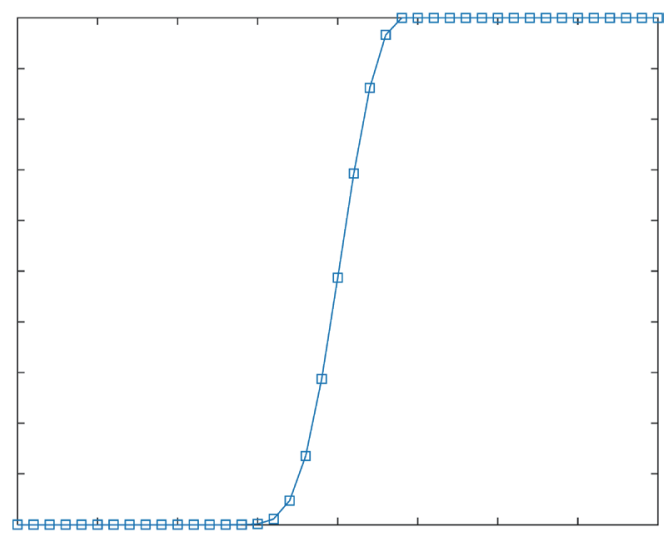

(b)

Fig. 3. (Color online) (a) Similarity detection of participating in DR and (b) users' requirement on distributed energy resources. 
can be divided into (1) renewable energy and (2) backup power of an alternator. According to the above situation, the following list is formed: (1) (participation, renewable energy); (2) (nonparticipation, renewable energy); (3) (participation, backup power of alternators); (4) (nonparticipation, reserved backup power of alternators), and the mathematical expression of the renewable energy distribution is as follows:

$$
S_{h}=\left[\begin{array}{c}
D R_{h} \times D R_{D G, h}^{\text {renew }} \\
D R_{h}^{\prime} \times D R_{D G, h}^{\text {renew }}
\end{array}\right]=\left[\begin{array}{c}
S_{h 1} \\
S_{h 2}
\end{array}\right]
$$

- Renewable energy distribution:

Participation strategy:

$$
S_{h, \text { renew }}=S_{D G, h} \times \frac{S_{h 1}}{\sum S_{h 1}+S_{h 3}} .
$$

Nonparticipation strategy:

$$
S_{h, \text { renew }}=S_{D G, h} \times \frac{S_{h 2}}{\sum S_{h 2}+S_{h 4}} .
$$

The threshold value of the DR is $50 \mathrm{~kW}$, and after the loads distributed to the renewable energy resource and actually turned off are deducted, $S_{\text {h,acgen }}$ is the resource needed to be supplemented by the generator.

- Power resource distribution of an alternator:

Participation strategy:

$$
S_{h, \text { acgen }}=50 \mathrm{~kW}-S_{h, \text { renew }}-S_{l, h}
$$

\subsection{Operation strategy study}

In the distributed energy dispatch strategy, factors such as the load variation, renewable energy variation, DR, and time-of-use power price are considered to analyze the users in different situations; to adjust users' energy storage equipment, diesel generators, and load control by using the estimated solar power generation; and to maximize the benefits of power consumption.

There are two operation strategies: the dispatch strategy of participation in DR and the dispatch strategy of nonparticipation in DR. In the strategy of participation in DR, participation in DR occurs when $D R_{h}>0.5$, with the process shown in Fig. 4 . The variable loads are adjusted to reduce power consumption during daily peak hours. By coordinating with the DR, if the renewable energy resources are insufficient, diesel generators and storage batteries are used 


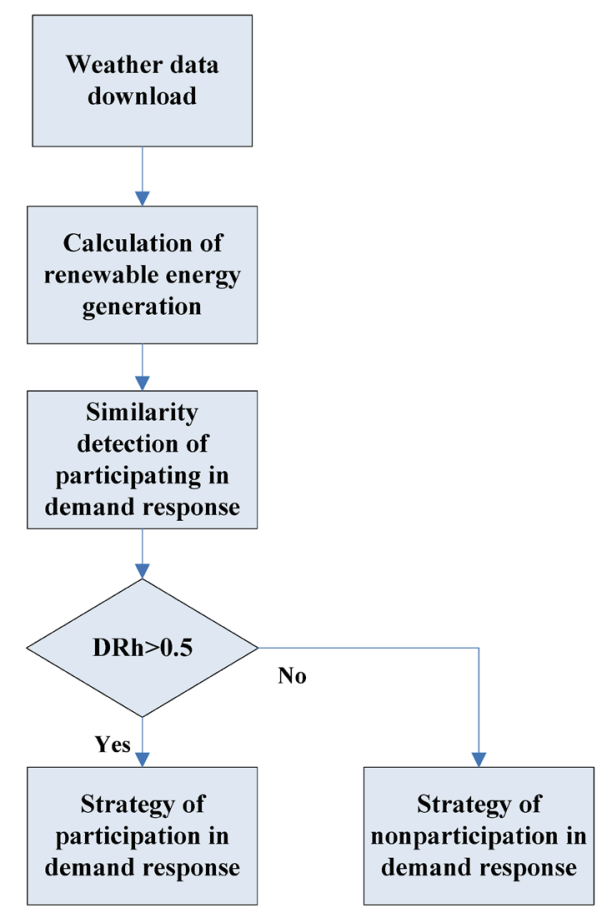

Fig. 4. (Color online) Decision-making process of participation in DR.

to meet the power demand. In the dispatch strategy of no DR, energy storage devices and diesel generators are mainly used to reduce the expenses of power users. In this paper, for the two strategies, the load curves of industrial power users were simulated using Taiwan Power Company's daily power consumption curve and the reliable power price table of DR. The peak hours from 10 am to $12 \mathrm{pm}$ and from $1 \mathrm{pm}$ to $3 \mathrm{pm}$ (a total of $4 \mathrm{~h}$ ) were selected for the DR, and various situations were simulated for actual measurement analysis to calculate the maximum benefits to be obtained.

\subsubsection{Strategies of participation and nonparticipation in DR}

(1) Strategy of nonparticipation in DR

The distributed energy of grids is a diverse system and sufficient reserve capacity must be provided, so nonparticipation in DR is chosen in the case of insufficient reserve capacity $\left(D R_{h}<0.5\right)$. In terms of power consumption, renewable energy (wind energy, solar energy) and stored energy are provided preferentially, and the distributed capacity of the renewable energy distribution capacity $S_{h, \text { renew }}$ and the distributed capacity of the alternator backup power $S_{h, \text { acgen }}$ are allocated according to DGM resources. The power consumption flow is shown in Fig. 5.

(2) Strategy of participation in DR

In the strategy of participation in DR, the distributed energy is dispatched during the peak hours from 10 am to $12 \mathrm{pm}$ and from $1 \mathrm{pm}$ to $3 \mathrm{pm}$. The power consumption flow is shown in Fig. 6. 


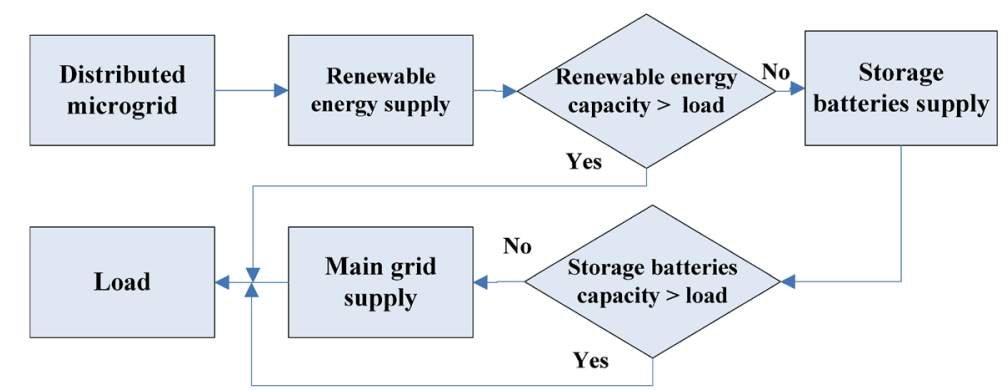

Fig. 5. (Color online) Decision-making process of strategy of nonparticipation in DR.



Fig. 6. (Color online) Decision-making process of strategy of participation in DR.

According to the DR management measures of Taiwan Power Company, the minimum limit of each low capacity is $50 \mathrm{~kW}$, so the following condition is met:

$$
S_{D G, h}+S_{i, h} \geq 50 \mathrm{~kW}
$$

According to the DR management measures of Taiwan Power Company, ${ }^{(11)}$ the notice for reducing power consumption is given a day in advance, and the quoted price per degree of reduced power consumption is submitted to the power company at application and is no more than $\$ 10$ (to 2 decimal places), and the change may be applied before $11 \mathrm{am}$ on the day before reducing power consumption. The daily notice to reduce power consumption can be $15 \mathrm{~min}$, $30 \mathrm{~min}, 1 \mathrm{~h}, 2 \mathrm{~h}$, or 1 day in advance. Different electricity charge deductions are provided at different time points, and the deduction standards are shown in Table 2.

\section{Results of Simulation Test}

In this study, it is assumed that a power consumer participated in DR with a reliable plan, a low contract capacity of $50 \mathrm{~kW}$, and a distributed energy system. The distributed energy included solar power, wind power, fuel cells, storage batteries, and backup diesel generators. The users' daily load curve in summer is shown in Fig. 7(a). The maximum demand was at 11 am (maximum demand $=93.7 \mathrm{~kW}$ ), and the solar power generation was sufficient from $10 \mathrm{am}$ to $3 \mathrm{pm}$. Hence, the peak hours ( $10 \mathrm{am}$ to $12 \mathrm{pm}$ and $1 \mathrm{pm}$ to $3 \mathrm{pm}$, a total of 4 h over two 
Table 2

Electricity charge deduction standards for implementation of reducing power consumption.

\begin{tabular}{lcc}
\hline $\begin{array}{l}\text { Notice of implementation of reducing } \\
\text { power consumption }\end{array}$ & $\begin{array}{c}\text { Basic electricity charge deduction } \\
(\$ / \mathrm{kW})\end{array}$ & $\begin{array}{c}\text { Current electricity charge deduction } \\
(\$ / \mathrm{kW})\end{array}$ \\
\hline $15 \mathrm{~min}$ & 65 & 10 \\
$30 \mathrm{~min}$ & 65 & 10 \\
$1 \mathrm{~h}$ & 65 & 8 \\
$2 \mathrm{~h}$ & 65 & 6 \\
Previous day & 65 & 4 \\
\hline
\end{tabular}

(1) Basic electricity charge deduction: reduced contract capacity $\times 65 \$ / \mathrm{kW} \times 120 \%$.

(2) Current electricity charge deduction: actual reduced capacity $\times$ hours of low power consumption implementation $\times$ quoted price per degree of lower power consumption.

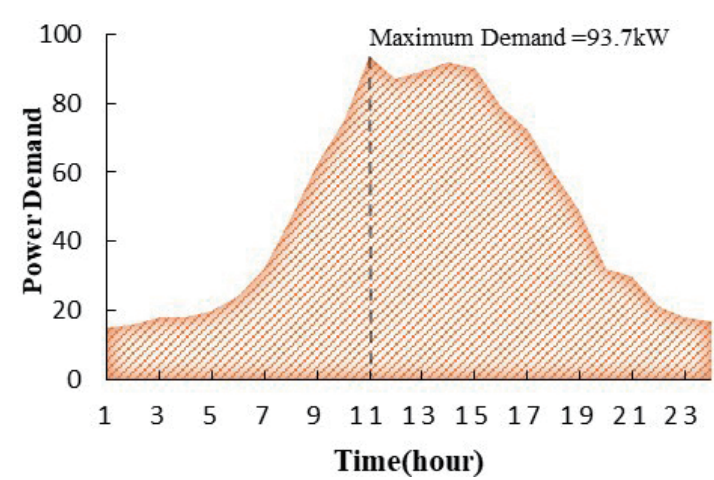

(a)

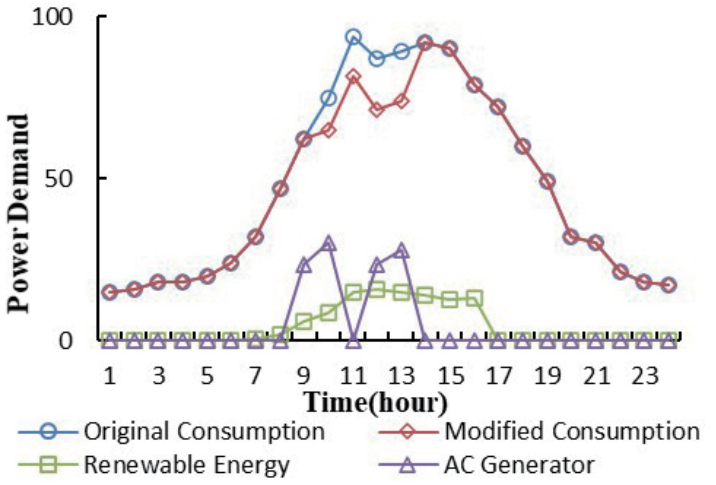

(b)

Fig. 7. (Color online) (a) Daily load curve of power users and (b) allocation results of DR and distributed energy resources.

periods) were selected as the DR time to effectively suppress the maximum demand. The load was reduced in cooperation with the disposable load of backup diesel generators and power users to reduce the demand on the main grid, and the power company was informed of the DR a day in advance.

As shown in Fig. 7(b) and Table 3, the hour from $12 \mathrm{pm}$ to $1 \mathrm{pm}$ is excluded from the $5 \mathrm{~h}$ from 10 am to $3 \mathrm{pm}$, the average hourly reduced capacity is $53 \mathrm{~kW}$, the reduced capacity is $50 \mathrm{~kW}$ more than the minimum limit, the renewable energy provides a total power of about $48 \mathrm{~kW}$ or an average of $12 \mathrm{~kW}$ per hour, the backup generator provides a total power of about $105 \mathrm{~kW}$ or an average of $26.24 \mathrm{~kW}$ per hour, and the average hourly power outage is $14.75 \mathrm{~kW}$. In terms of this DR, according to the electricity charge deduction standards in Table 2, a total of electricity charge deduction of NT\$ 4732, comprising the basic electricity charge of 3900 and the current electricity charge of 832 , is obtained. The electricity charge deduction incentive in all periods is shown in Table 4.

Without considering the brands and electrical loads of backup diesel generators, the generator fuel consumption was $7.5 \mathrm{l} / \mathrm{h}$, the fuel consumption of power generation in $4 \mathrm{~h}$ was about $30 \ell$, the diesel was priced at 15 per liter, and the cost of the backup diesel generators was 
Table 3

Allocation results of DR and distributed energy resources.

\begin{tabular}{lccccccc}
\hline Time & $\boldsymbol{D R}_{\boldsymbol{h}}$ & $\boldsymbol{D R}_{\boldsymbol{h}}{ }^{\prime}$ & $\boldsymbol{D R}_{\boldsymbol{D G}, \boldsymbol{h}}^{\text {renew }}$ & $\boldsymbol{S}_{\boldsymbol{h} \mathbf{1}}$ & $\boldsymbol{S}_{\boldsymbol{h} \mathbf{2}}$ & $\begin{array}{c}\text { Renewable } \\
\text { energy (kW) }\end{array}$ & $\begin{array}{c}\text { AC generator } \\
(\mathrm{kW})\end{array}$ \\
\hline 10:00-11:00 & 0.7742 & 0.2258 & 0.8735 & 0.6763 & 0.1972 & 11.6132 & 23.3868 \\
11:00-12:00 & 0.8584 & 0.1416 & 0.6869 & 0.5896 & 0.0973 & 12.8754 & 30.1246 \\
13:00-14:00 & 0.7955 & 0.2045 & 0.9187 & 0.7309 & 0.1878 & 13.5242 & 23.4758 \\
14:00-15:00 & 0.8334 & 0.1666 & 0.946 & 0.7883 & 0.1576 & 10.0002 & 27.9998 \\
\hline & & & & & Total & 48.013 & 104.987 \\
\hline
\end{tabular}

Table 4

Results of electricity charge deduction.

\begin{tabular}{lcc}
\hline $\begin{array}{l}\text { Notice of implementation of reducing } \\
\text { power consumption }\end{array}$ & $\begin{array}{c}\text { Basic electricity charge deduction } \\
(\$ / \mathrm{kW})\end{array}$ & $\begin{array}{c}\text { Current electricity charge deduction } \\
(\$ / \mathrm{kW})\end{array}$ \\
\hline $15 \mathrm{~min}$ & $50 \times 65 \times 1.2=3900$ & $52 \times 4 \times 10=2080$ \\
$30 \mathrm{~min}$ & $50 \times 65 \times 1.2=3900$ & $52 \times 4 \times 10=2080$ \\
$1 \mathrm{~h}$ & $50 \times 65 \times 1.2=3900$ & $52 \times 4 \times 8=1664$ \\
$2 \mathrm{~h}$ & $50 \times 65 \times 1.2=3900$ & $52 \times 4 \times 6=1248$ \\
Previous day & $50 \times 65 \times 1.2=3900$ & $52 \times 4 \times 4=832$ \\
\hline
\end{tabular}

about $30 \times 15=450$. Hence, after the power generation cost was deducted from the electricity charge, an electricity charge deduction incentive of 4282 was obtained in this implementation of DR. If a total of nine DRs in a month were implemented, a deduction incentive of $4282 \times 9$ $=38538$ would be obtained. However, all DRs had different results, so it was estimated that the deduction incentive for one month ranged from 35000 to 40000.

\section{Discussion}

The results of the simulation test verified the use of the DGM to determine whether to participate in DR according to the power outage, distributed energy capacity, and backup generator capacity, and to allocate the renewable energy, the capacity of backup diesel generators, and detachable loads required to participate in DR during peak hours, so as to maintain low power consumption below $50 \mathrm{~kW}$. The results show that the DGM can effectively distribute power and achieve the optimal results for power consumption and return.

\section{Conclusions}

According to the research results of this study, the DR plan was found to be reliable, and reducing power consumption was implemented for $36 \mathrm{~h}$ in one month. If the contract conditions for reducing power were met, the incentive of electricity charge deduction after implementation would be about NT\$ 35000-40000.

If users can expand the distributed power generation capacity and storage capacity and cooperate with government programs, such as the green roof program with civic participation ${ }^{(12)}$ and the subsidized solar photovoltaic power generation system implementation program, ${ }^{(13-15)}$ to gain more benefits, and if the power company provides more effective strategies, such as a 
higher electricity charge discount, off-peak electricity for air conditioning systems, and power consumption reduction, to effectively reduce the power consumption during peak hours, then, under this architecture, carbon emissions can be effectively reduced, the power industry's reserve capacity can be increased, and users can also obtain cheaper electricity, resulting in a win-win-win situation.

\section{References}

1 N. Lu: IEEE Trans. Smart Grid 3 (2012) 1263.

2 L. Gkatzikis, I. Koutsopoulos, and T. Salonidis: IEEE J. Sel. Areas Commun. 31 (2013) 1247. https://doi. org/10.1109/JSAC.2013.130708

3 C. Y. Hsu and R. C Wu: 2014 Economic Construction Seminar (ECS, 2014) 59-83. http://readopac1.ncl.edu. tw/nclJournal/search/detail.jsp?sysId=0006778526\&dtdId=000040\&search type $=$ detail\&la $=\mathrm{ch}$

4 K. C. Lu, R. W. Huang, H. M. Lin, and S. Y. Lin: 12th Annu. China Association for Science and Technology (2010). http://d.g.wanfangdata.com.hk/Conference 7333495.aspx

5 L. Y. Chang, Y. N. Chung, H. S. Chen, S. L. Kuo, and C. H. Lin: Apply Dynamic Game Model to Activate Distributed Generations for an Aggregator Model in Smart Grids (Taiwan Power Electronics Association, 2017). http://readopac2.ncl.edu.tw/nclJournal/search/detail.jsp?sysId=0006921176\&dtdId=000040\&search type $=$ detail\&la $=$ ch

6 H. S. Chen: Conf. Applications of Innovation and Invention \& The 24th National Conf. Fuzzy Theory and Its Applications (November 2016).

7 L. Zhao, Y. B. Liu, L. N. Yu, and J. Y. Liu: Power System Protection and Control Conf. (October 2019).

8 M. L. Chen: Short-Term Prediction of Wind Power and Application (Taiwan Electric Engineering Monthly, 2007). https://www.twtpo.org.tw/upload/201409/28/201409280945535312.pdf

9 M. A. Nowak: Science: Five Rules for the Evolution of Cooperation (December 2006) 1560-1563.

10 J. B. Kadane and P. D. Larkey: Manag. Sci. 28 (1982) 113. https://doi.org/10.1287/mnsc.28.2.113

11 Taiwan Power Company: Load Management Measures of Demand Response, https://www.taipower.com.tw/ upload/135/2018071708513325281.pdf (accessed July 2018).

12 Executive Yuan World Information Network: Green Roof Program with Civic Participation, https://www. ey.gov.tw/Page/5A8A0CB5B41DA11E/e9cb7d49-3982-4f9d-8cfe-8f7096df3acc (accessed January 2019).

13 Economic Development Bureau, Taichung City Government: Solar Photovoltaic Power Generation System Implementation Program with Subsidies, https://www.economic.taichung.gov.tw/1261663/post (accessed January 2020).

14 J. R. Molina, M. M. Nunez, J. F. Martínez, and W. P. Aguiar: Energies 7 (2014) 6142. https://doi.org/10.3390/ en7096142

15 Oleg Gulich: Technological and Business Challenges of Smart Grids-aggregator's Role in Current Electricity Market, Master's Thesis, Lappeenranta University of Technology, Faculty of Technology and Electrical Engineering (2010).

\section{About the Authors}

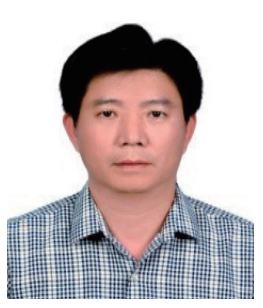

Long-Yi Chang received his B.S. degree in electrical engineering from Chin-Yi Institute of Technology and Commerce, Taichung, Taiwan, in 1992 and his M.S. degree in automatic control engineering from Feng Chia University, Taichung, Taiwan, in 2003. He received his Ph.D. degree in electrical engineering from National Changhua University of Education, Changhua, Taiwan, in 2018. He is presently an associate professor at the National Chin-Yi University of Technology, Taichung, Taiwan. His areas of interest include distribution system design, renewable energy, electrical energy management, and energy-conservation technology.

(lychang@ncut.edu.tw) 


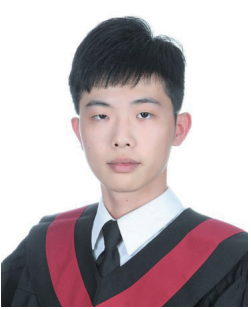

Shiu-Fu Lin was born in Taichung, Taiwan, in 1996. He received his B.S. degree from the Department of Electrical Engineering, Chin-Yi University of Technology, Taichung, Taiwan, in 2018. He is currently a graduate student in the Department of Electrical Engineering of National Chin-Yi University of Technology. His areas of interest include renewable energy, electrical energy management, and energy-conservation technology. (linjerry56@gmail.com) 